\title{
Distributed chaos of the atmospheric tracers
}

\author{
A. Bershadskii \\ ICAR, P.O. Box 31155, Jerusalem 91000, Israel \\ bershads@gmail.com
}

\begin{abstract}
It is shown, using results of numerical simulations, atmospheric experiments and observations with natural trace-gases (ozone, carbon dioxide etc.), that the spatio-temporal distributed chaos can provide good quantitative description of the statistical properties of the passive scalar tracers in the atmosphere from the atmospheric surface layer up to the stratosphere. Related properties of the solar radiation transmittance in the atmosphere have been also briefly discussed as well as analogous properties of the tracers in Jupiter's atmosphere.
\end{abstract}

\section{DETERMINISTIC CHAOS}

It is known that the chaotic smooth dynamical systems with compact strange attractors usually have exponential frequency spectra 1]-[7]. For the systems described by the equations with partial derivatives one can expect that the spatio-temporal smoothness will also result in the wavenumber exponential spectra.

The dynamics of a passive scalar tracer $\chi$ in a velocity field $\mathbf{u}$ can be described by equation

$$
\partial_{t} \chi+\mathbf{u} \cdot \nabla \chi=\kappa \nabla^{2} \chi+\mathbf{f}_{\chi}
$$

Results of a direct numerical simulation of the passive scalar tracers mixing in the statistically stationary isotropic homogeneous fluid motion described by the incompressible Navier-Stokes equations

$$
\begin{gathered}
\partial_{t} \mathbf{u}+\mathbf{u} \cdot \nabla \mathbf{u}=-\nabla p+\nu \nabla^{2} \mathbf{v}+\mathbf{f} \\
\nabla \cdot \mathbf{u}=\mathbf{0}
\end{gathered}
$$

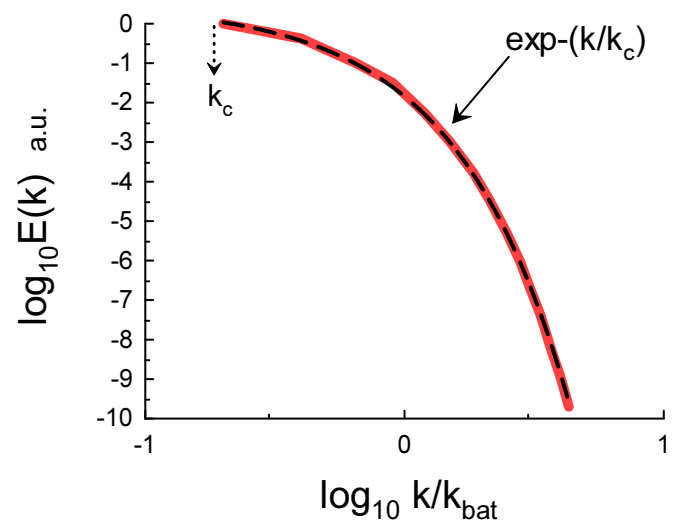

FIG. 1: Power spectrum of passive scalar fluctuations for isotropic homogeneous (steady) spatio-temporal fluid chaos $\left(R e_{\lambda}=8\right.$ and $\left.S c=1\right)$. for small Reynolds numbers were reported in the paper Ref. 8].

Figure 1 shows the wavenumber power spectra for such passive scalar tracer mixing for the Taylor-Reynolds number $R e_{\lambda}=8$ (the Schmidt number $S c=1$ and the periodic boundary conditions were used). For such small Reynolds number the passive scalar concentration field $\chi$ can be represented by a deterministic chaos and has an exponential wavenumber power spectrum

$$
E(k) \propto \exp -\left(k / k_{c}\right)
$$

The position of the characteristic wavenumber $k_{c}$ is indicated in the Fig. 1 by the dotted arrow, $k_{b a t}$ is the Batchelor wavenumber and the dashed curve is drawn to indicate the exponential type of the spectrum. The spectral data for the Fig. 1 were taken from Fig. 1a of the Ref. 8] (see the Ref. [8 for more detail description of the numerical simulation).

\section{BUOYANCY-DRIVEN THERMAL CONVECTION}

The buoyancy-driven thermal convection in the Boussinesq approximation can be described by equations 9]

$$
\begin{gathered}
\frac{\partial \mathbf{u}}{\partial t}+(\mathbf{u} \cdot \nabla) \mathbf{u}=-\frac{\nabla p}{\rho_{0}}+\sigma g \theta \mathbf{e}_{z}+\nu \nabla^{2} \mathbf{u}+\mathbf{f} \\
\frac{\partial \theta}{\partial t}+(\mathbf{u} \cdot \nabla) \theta=S \frac{\Delta}{H} e_{z} u_{z}+\kappa \nabla^{2} \theta \\
\nabla \cdot \mathbf{u}=\mathbf{0}
\end{gathered}
$$

where $\theta$ is the temperature fluctuations over an imposed temperature profile $T_{0}(z), \mathbf{e}_{z}$ is a unit vector along the gravity force, $g$ is the gravity acceleration, $\Delta$ is the temperature difference between the different layers, $H$ is the distance between the layers, $\rho_{0}$ is the mean density, $\sigma$ is the thermal expansion coefficient, $\nu$ and $\kappa$ are the viscosity and thermal diffusivity, $\mathbf{f}$ is an 


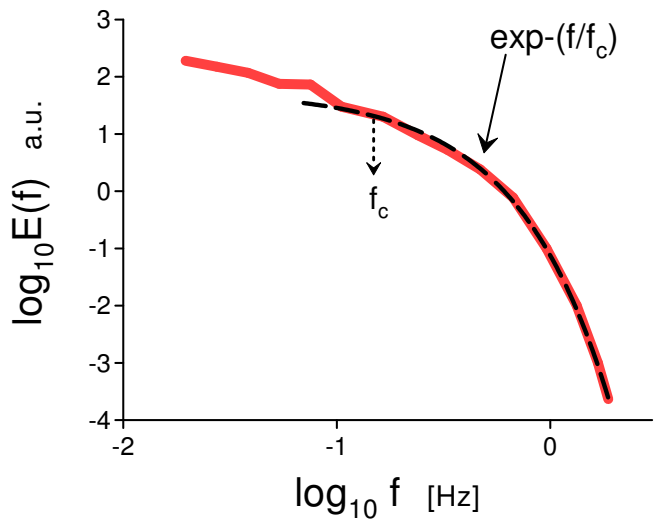

FIG. 2: Power spectrum of the concentration fluctuations for the passive scalar tracers ejected from a line source into the atmospheric surface layer at stable conditions.

external force. For the unstable stratification the parameter $S=+1$ and for the stable stratification it is $S=-1$.

For the inviscid case (when $R a \rightarrow \infty$ ) the equation for the mean helicity is

$$
\frac{d\langle h\rangle}{d t}=2\langle\boldsymbol{\omega} \cdot \mathbf{F}\rangle
$$

with

$$
\mathbf{F}=(\sigma g \theta) \mathbf{e}_{z}+\mathbf{f},
$$

here $\boldsymbol{\omega}=\nabla \times \mathbf{u}$ and $h=\mathbf{u} \cdot \boldsymbol{\omega}$ are the vorticity and helicity fields respectively, and $\langle\ldots\rangle$ is an average over the liquid volume. It is clear from the Eq. (8) that the mean helicity is not an inviscid invariant for this case or it is equal to zero due to a global spatial symmetry. We will consider, therefore, the case when the large-scale forces only will contribute the main part to the correlation $\langle\boldsymbol{\omega} \cdot \mathbf{F}\rangle$. The correlation $\langle\boldsymbol{\omega} \cdot \mathbf{F}\rangle$, however, is rapidly decreasing with spatial scales (such situation is typical for the highly chaotic flows). Therefore, in spite of the mean helicity is not an inviscid invariant here the higher moments of the helicity distribution $h=\mathbf{u} \cdot \boldsymbol{\omega}$ can be still (approximately) considered as the inviscid invariants [10], 11].

To show this, we will divide the liquid volume into a net of the cells which are moving with the liquid (the Lagrangian description) $-V_{i}[10] 11$. The boundary conditions on their surfaces are taken as $\boldsymbol{\omega} \cdot \mathbf{n}=0$. Moments of order $n$ can be defined as

$$
I_{n}=\lim _{V \rightarrow \infty} \frac{1}{V} \sum_{j} H_{j}^{n}
$$

where the total helicity $H_{j}$ in the subvolume $V_{j}$ is

$$
H_{j}=\int_{V_{j}} h(\mathbf{r}, t) d \mathbf{r} .
$$

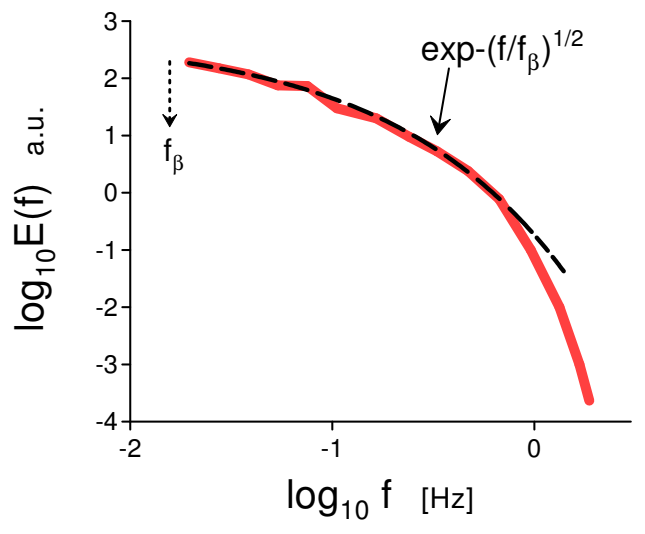

FIG. 3: As in the Fig. 2 but the dashed curve indicates the stretched exponential spectral law Eq. (15) for the small $f$.

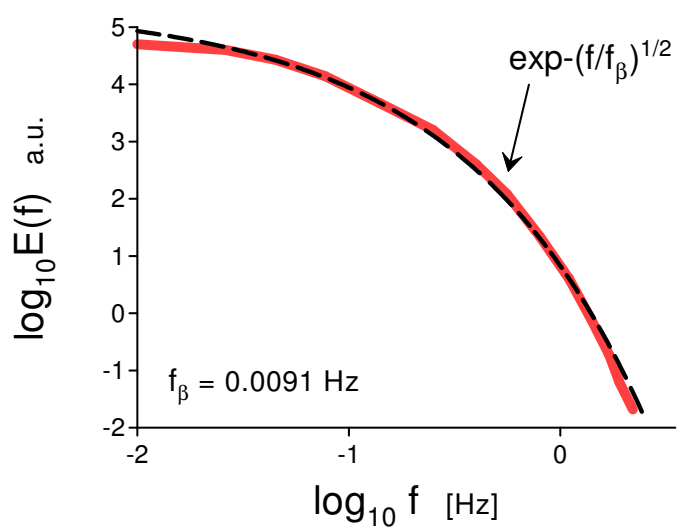

FIG. 4: Power spectrum of the concentration fluctuations for the passive scalar tracers ejected from a linear source in the atmospheric surface layer over a homogeneous canopy at mildly unstable to very unstable conditions.

Due to the fast reduction of the correlation $\langle\boldsymbol{\omega} \cdot \mathbf{F}\rangle$ with the scales the helicities $H_{j}$ can be approximately considered as inviscid invariants for the cells with the small enough characteristic spatial scales. Just these cells provide the main contribution to the moments $I_{n}$ with $n \gg 1$ for the chaotic flows (cf. [12]). Therefore, the moments $I_{n}$ for the sufficiently large $n$ can be considered as inviscid quasi-invariants while the total helicity $I_{1}$ cannot. For the strongly chaotic flows the values $n=2$ and $n=3$ can be still considered as sufficiently large (where the moment $I_{2}$ is the socalled Levich-Tsinober invariant of the Euler equation [10]). For the viscous cases such moments can be considered as adiabatic invariants in the inertial range of scales.

The chaotic attractors (in the phase space) correspond 


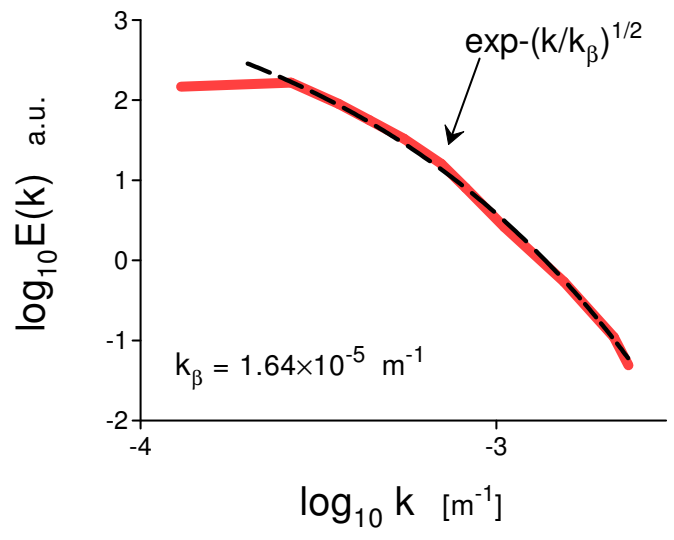

FIG. 5: Power spectrum of the ozone mixing ratio fluctuations in the lower stratosphere.

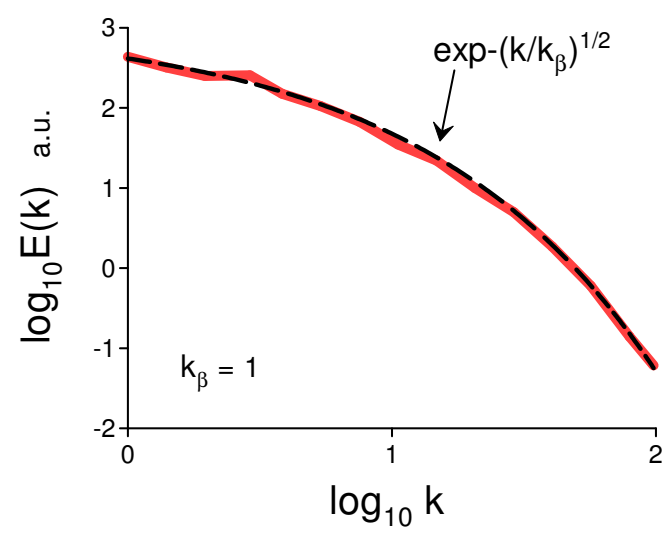

FIG. 6: Power spectrum of the ozone mixing ratio fluctuations in the global upper troposphere and lower stratosphere at $55^{\circ} \mathrm{N}$.

to the adiabatic invariants $I_{n}$. Their basins of attraction, however, can be significantly different: the attractors corresponding to the larger $n$ have thinner basin of attraction (the intermittency). Therefore, the flow dynamics is dominated by the invariant $I_{n}$ with the smallest (available) order $n$.

We will begin consideration from $I_{3}$, for simplicity. The dimensional considerations can be used to estimate characteristic velocity $u_{c}$ for the fluctuating $k_{c}$

$$
u_{c} \propto\left|I_{3}\right|^{1 / 6} k_{c}^{1 / 2}
$$

in this case

\section{HELICAL DISTRIBUTED CHAOS}

Figure 2 shows power spectrum of the concentration fluctuations for the passive scalar tracers (sulfur hexafluoride - $S F_{6}$ ) ejected from a line source into the atmospheric surface layer at stable conditions. The spectral data were taken from Fig. 1 of the Ref. [13. This is a frequency $(E(f))$ spectrum obtained from the time series of the passive scalar concentration measured by a probe. The frequency spectrum represents actually the spatial structures passing through the probe with the mean velocity of the wind $U_{0}$ and the frequency spectrum should be converted into a wavenumber spectrum using the Taylor's 'frozen' turbulence hypothesis [14] with $f=U_{0} k / 2 \pi$, where $k$ is the wavenumber. Therefore, the dashed curve is drawn in the Fig. 2 to indicate the exponential spectral law Eq. (4). This spectral law gives a good approximation for the large frequencies, i.e. for the large wavenumbers where the diffusivity (viscosity) at the moderately stable conditions provides existence of a deterministic chaos. For the small wavenumbers, however, the influence of the diffusive processes is not strong enough and the parameter $k_{c}$ in the Eq. (4) becomes fluctuating. These fluctuations can be taken into account with an ensemble average

$$
E(k) \propto \int_{0}^{\infty} P\left(k_{c}\right) \exp -\left(k / k_{c}\right) d k_{c}
$$

here $P\left(k_{c}\right)$ is the probability distribution of the characteristic scale $k_{c}$.

Assuming a Gaussian (normal) distribution of the characteristic velocity $u_{c}$ [14, one can obtain the distribution $P\left(k_{c}\right)$ for the helically dominated distributed chaos from the Eq. (12), for instance,

$$
P\left(k_{c}\right) \propto k_{c}^{-1 / 2} \exp -\left(k_{c} / 4 k_{\beta}\right)
$$

where the new parameter $k_{\beta}$ is a constant (see below).

Substituting the Eq. (14) into the Eq. (13) one obtains

$$
E(k) \propto \exp -\left(k / k_{\beta}\right)^{1 / 2}
$$

for the helically dominated distributed chaos.

Figure 3 shows power spectrum of the concentration fluctuations for the passive scalar tracers as it is shown in the Fig. 2 but now the dashed curve indicates the stretched exponential spectral law Eq. (15) for the small values of the wavenumber $k$.

Figure 4 shows power spectrum of the concentration fluctuations for the passive scalar tracers over a homogeneous canopy (a peach orchard) of intermediate roughness ejected from a line source in the atmospheric surface layer at mildly unstable to very unstable conditions. The spectral data were taken from Fig. $2 \mathrm{~b}$ of the Ref. 15] (the spectrum was computed using 


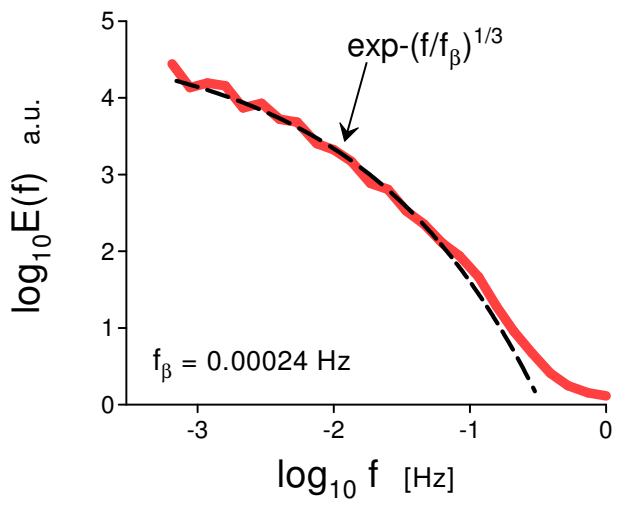

FIG. 7: Power spectrum of the of the naturally emitted nitric oxide (NO) within an Amazonian rain forest at the height $1 \mathrm{~m}$ above the forest floor.

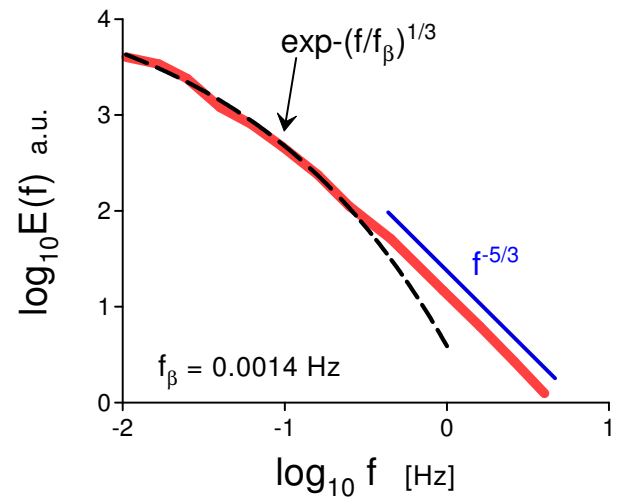

FIG. 8: Power spectrum of the $\mathrm{CO}_{2}$ concentration fluctuations measured in the atmospheric surface layer over an abandoned peat meadow with comparatively high $\mathrm{CO}_{2}$ uptake.

Yule-Walker AR method). The dashed curve indicates the stretched exponential spectral law Eq. (15)

The results of the 4 year measurements made with an ozonesonde in the lower stratosphere over a latitudinal region $60^{\circ} \mathrm{S}-43^{\circ} \mathrm{N}$ were reported in the paper Ref. [16]. A research oceanic vessel was used as a main base. The measurements were made in the altitude range $19-27 \mathrm{~km}$.

Figure 5 shows power spectrum of the ozone mixing ratio fluctuations (averaged over all measurements). The spectral data were taken from the Fig. 5 of the Ref. [16]. The dashed curve is drawn to indicate the stretched exponential spectrum Eq. (15).

Figure 6 shows power spectrum of the ozone distribution obtained at $55^{\circ} \mathrm{N}$ in February 2005 (the spectral data were taken from Fig. 4b of the paper Ref. [17]). The assimilation in the upper troposphere and lower stratosphere was studied with the NASA's EOS-Aura ozone data using meteorological fields from the Goddard Earth Observing System. The dashed curve is drawn to indicate the stretched exponential spectrum Eq. (15). The wavenumber $k_{\beta}=1$ corresponds to the longest planetary wave and, as it follows from the Fig. 6, the entire helical distributed chaos is tuned to the longest planetary wave.

\section{STRONG DISTRIBUTED CHAOS}

It is not surprising that for the smooth dynamical systems the spectrum has the stretched exponential form. The particular stretched exponential Eq. (15) can be generalized

$$
E(k) \propto \exp -\left(k / k_{\beta}\right)^{\beta},
$$

where the $k_{\beta}$ is a constant.

In order to find value of the $\beta$ we can use asymptotic (at $k_{c} \rightarrow \infty$ ) properties of the distribution $P\left(k_{c}\right)$. It follows from the Eqs. (13) and (16) at this asymptotic [18.

$$
P\left(k_{c}\right) \propto k_{c}^{-1+\beta /[2(1-\beta)]} \exp \left(-b k_{c}^{\beta /(1-\beta)}\right),
$$

with $b$ as a constant. On the other hand, the asymptotic distribution $P\left(k_{c}\right)$ can be found from the dimensional considerations. Indeed, the particular estimate Eq. (12) can be replaced by a general estimate

$$
u_{c} \propto\left|I_{n}\right|^{1 / 2 n} k_{c}^{\alpha_{n}}
$$

where

$$
\alpha_{n}=1-\frac{3}{2 n}
$$

If again $u_{c}$ has Gaussian (normal) distribution a relationship between the exponents $\beta_{n}$ and $\alpha_{n}$ can be readily obtained from the Eqs. (17) and (18)

$$
\beta_{n}=\frac{2 \alpha_{n}}{1+2 \alpha_{n}}
$$

Substituting $\alpha_{n}$ from the Eq. (19) into the Eq. (20) we obtain

$$
\beta_{n}=\frac{2 n-3}{3 n-3}
$$

For strongly chaotic helicity field even value $n=2$ can be considered as sufficiently large to consider the second moment $I_{2}$ (the Levich-Tsinober invariant of the Euler equation [10, 11]) as an adiabatic invariant (see Section III). In this case we obtain from the Eq. (21) $\beta=1 / 3$, i.e.

$$
E(k) \propto \exp -\left(k / k_{\beta}\right)^{1 / 3}
$$




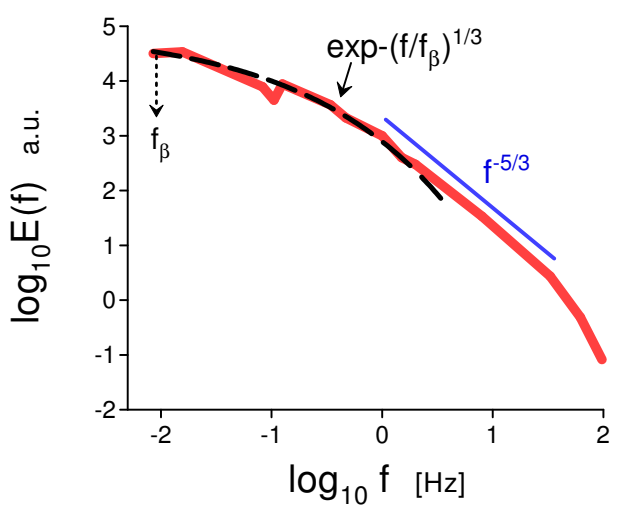

FIG. 9: Power spectrum of a passive scalar tracer $\left(\mathrm{C}_{3} \mathrm{H}_{6}\right)$ in a dispersing plume artificially ejected from an elevated point source in the atmospheric surface layer over a flat and smooth area at near-neutral stability conditions.

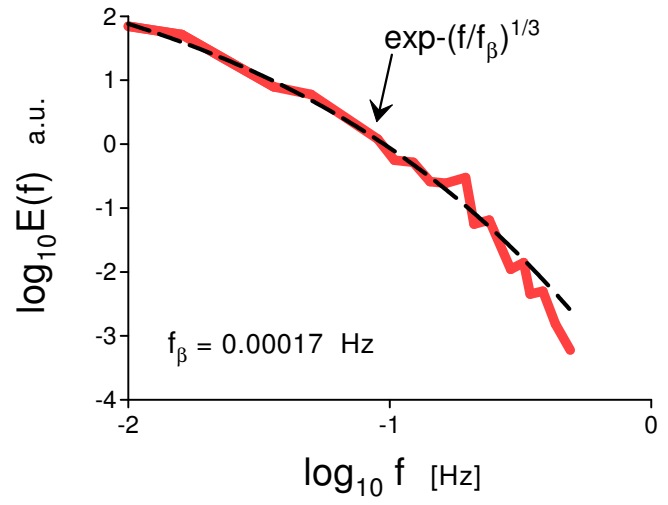

FIG. 10: Power spectrum of the ozone concentration fluctuations in the anvil of a large cumulonimbus cloud (of a severe thunderstorm).

Figure 7 shows power spectrum of the naturally emitted nitric oxide (NO) within an Amazonian rain forest at the height $1 \mathrm{~m}$ above the forest floor. The spectral data were taken from the Fig. 3 of the Ref. [19]. The dashed curve indicates the stretched exponential spectral law Eq. (22).

The wet peat lands are considered as a sink of $\mathrm{CO}_{2}$. Figure 8 shows power spectrum of the $\mathrm{CO}_{2}$ concentration fluctuations measured over an abandoned peat meadow in the Netherlands with comparatively high $\mathrm{CO}_{2}$ uptake (the spectral data were taken from the Fig. 2 of the Ref.[20]). The measurement were performed in the atmospheric surface layer. The dashed curve indicates the stretched exponential spectral law Eq. (22).

Figure 9 shows power spectrum of a passive scalar tracer $\left(\mathrm{C}_{3} \mathrm{H}_{6}\right)$ in a dispersing plume artificially ejected from an elevated point source in the atmospheric surface

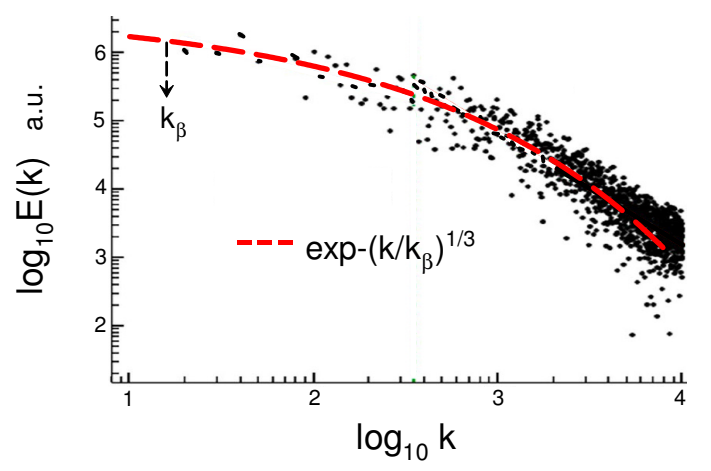

FIG. 11: Spectrum of the intensity fluctuations of the Jupiter's upper cloud layer reflectivity.

layer (at a release height of $2.5 \mathrm{~m}$ ). The experimental site was flat and smooth. The passive scalar tracer was released isotropically (without forming a jet). The measurements were performed near the mean-plume centerline under near-neutral stability conditions (the spectral data were taken from the Fig. 19 of the Ref. 21]). The dashed curve indicates the stretched exponential spectral law Eq. (22).

Figure 10 shows power spectrum of the ozone concentration fluctuations in the anvil of a large cumulonimbus cloud (of a severe thunderstorm). The spectral (aircraft-measured) data were taken from the Fig. 7 of the Ref. 22]. The measurements were performed in the convectively neutral region of the anvil, corresponding approximately to the altitude of the midsection of the thunderstorm core. The dashed curve indicates the stretched exponential spectral law Eq. (22).

\section{JUPITER'S UPPER CLOUD LAYER}

The Jupiter's upper atmosphere is characterized by banded continual appearance of the dark and light clouds (see, for instance, Ref. 23] and references therein). Complete maps of Jupiter's upper cloud layer obtained using the Hubble Space Telescope (at optical wavelengths) were reported in recent Ref. 24]. The observed global Jupiter's maps were then used in the Ref. 24] in order to compute corresponding passive scalar trasers' power spectra for single rotation observations. An example of the spectrum of the intensity fluctuations of the cloud's reflectivity is plotted in the Fig. 11 against the longitudinal wave number $k$ (which represents the number of complete sinusoids fitting in the circumference of the planet at a chosen latitude). The spectral data were taken from Fig. 3 of the Ref. 24. The dashed curve in the Fig. 11 indicates correspondence to the spectrum Eq. (22). 


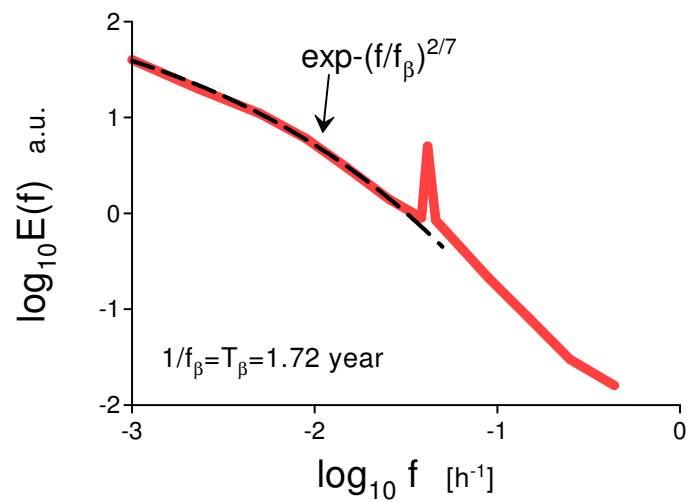

FIG. 12: Power spectrum of the atmospheric $\mathrm{CO}_{2}$ temporal fluctuations - high latitude (northern hemisphere).

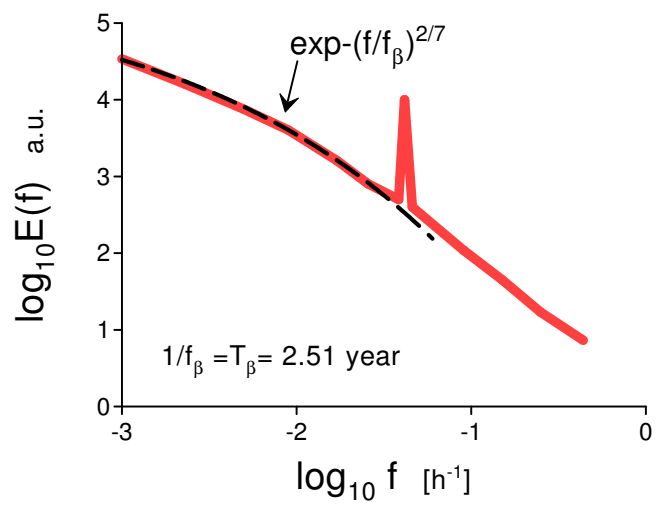

FIG. 13: Power spectrum of the atmospheric $\mathrm{CO}_{2}$ temporal fluctuations - middle latitude (northern hemisphere).

\section{TEMPORAL DISTRIBUTED CHAOS}

In the previous sections the spatial distributed chaos has been studied and the observed frequency spectra were a direct reflection of the spatial chaotic processes due to the Taylor's "frozen" fluctuations mechanism. In order to observe real temporal fluctuations one needs in measurements performed for larger temporal scales (see below).

Let us consider the temporal distributed chaos. In this case the spatial (wavenumber) Eqs. (13) and (16) should be replaced by temporal (frequency) equation

$$
E(f) \propto \int_{0}^{\infty} P\left(f_{c}\right) \exp -\left(f / f_{c}\right) d f_{c} \propto \exp -\left(f / f_{\beta}\right)^{\beta},
$$

Instead of the Eqs. (18) and (19) one obtains from the dimensional considerations

$$
u_{c} \propto\left|I_{n}\right|^{1 /(4 n-3)} f_{c}^{\alpha_{n}}
$$

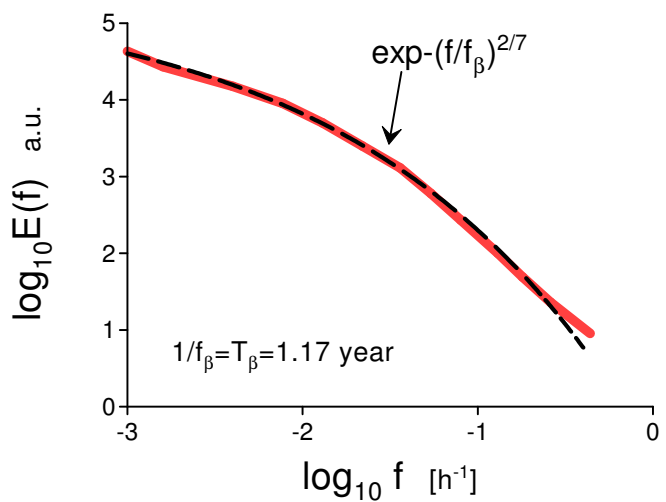

FIG. 14: Power spectrum of the atmospheric $\mathrm{CO}_{2}$ temporal fluctuations - low latitude (northern hemisphere).

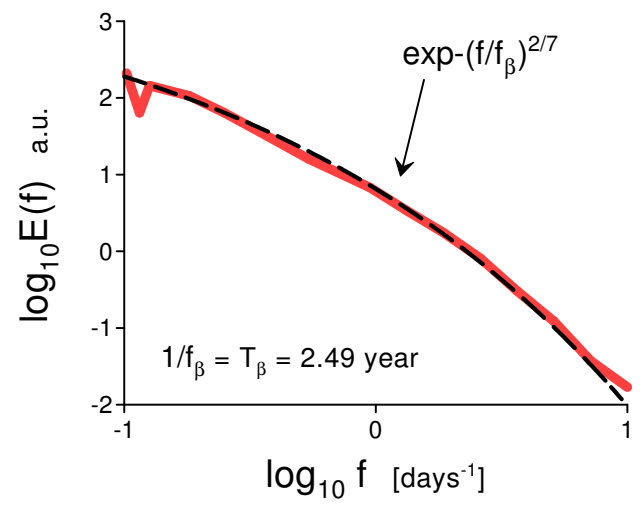

FIG. 15: Power spectrum of the atmospheric radon $\left({ }^{222} R n\right)$ temporal fluctuations - middle latitude (southern hemisphere).

where

$$
\alpha_{n}=\frac{2 n-3}{4 n-3}
$$

and instead of the Eq. (21)

$$
\beta_{n}=\frac{2(2 n-3)}{(8 n-9)}
$$

Then for the strongly chaotic helical distributed chaos $(n=2)$ one obtains $\beta=2 / 7$, i.e.

$$
E(f) \propto \exp -\left(f / f_{\beta}\right)^{2 / 7}
$$

for the proper temporal (frequency) spectrum.

Figures 12-14 show power spectra of the atmospheric $\mathrm{CO}_{2}$ temporal fluctuations measured in the atmospheric surface layer at the meteorological stations Barrow, 


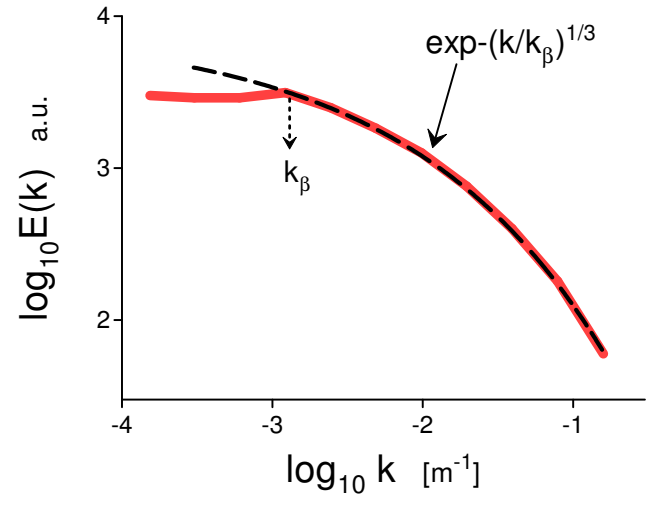

FIG. 16: Power spectrum of the global radiation transmittance (vs. the horizontal wavenumbers $k$ ) for the broken-cloud sky conditions.

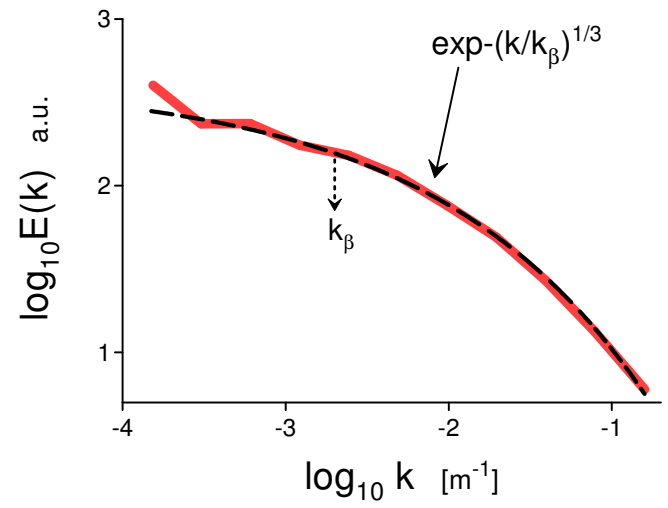

FIG. 17: As in the Fig. 16 but for the cirrus sky conditions.

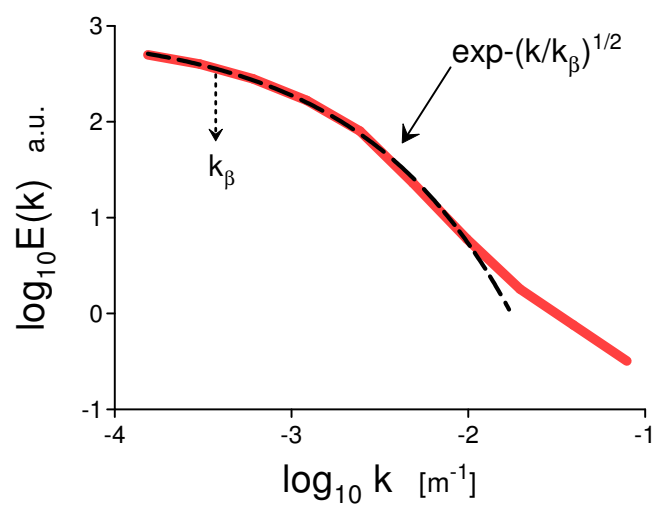

FIG. 18: As in the Fig. 16 but for the overcast cloudy sky conditions.
USA (high latitude), Schauinsland, Germany (middle latitude), and Hateruma, Japan (low lattidude), respectively (all three stations are located in the northern hemisphere). The spectral data were taken from the Fig. 2b of the Ref. 25. The peaks in the Figs. 12 and 13 correspond to the diurnal (24 hours) periodic variability. The dashed curves in the Figs. 12-14 indicate correspondence to the spectrum Eq. (27).

Figure 15 shows power spectrum of the atmospheric radon ( $\left.{ }^{222} R n\right)$ temporal fluctuations measured in the atmospheric surface layer at the Cape Point station (South Africa). This is a middle latitude coastal station in the Southern Hemisphere. The spectral data were taken from the Fig. 4 of the Ref. 26. The dashed curve in the Fig. 15 indicate correspondence to the spectrum Eq. (27). It should be noted that $T_{\beta}$ are about the same $(\simeq 2.5$ year $)$ for the middle latitude northern and southern hemispheres' observations (cf. Figs. 13 and 15).

\section{SOLAR RADIATION TRANSMITTANCE}

Properties of the atmospheric solar radiation transmittance are closely related to the properties of the atmospheric tracers, especially in presence of the clouds. The optical properties of the clouds are determined by their composition and its chaotic/turbulent dynamics. Generally, the droplets, aerosols etc. cannot be considered as dynamically passive scalars in this case. However, taking into account the above suggested separation of the scales for the helicity effects one can expect that the above consideration can be (with certain restrictions) applied to this case as well.

In the recent paper Ref. [27] results of measurements of the global radiation, performed using a ground-based (surface) dense network of 99 pyranometers for different sky conditions, were reported. The global transmittance was computed by normalizing the global radiation by the extraterrestrial radiation at the top of atmosphere and by accounting for the Sun-Earth distance and for the cosine of solar zenith angle. The experimental site $(10 \times 12 \mathrm{~km})$ was located near Julich (Germany) and the measurements were produced from April 2 to July 24, 2013 during the daylight periods. Transformation from frequency to wavenumber space was made using the Taylor "frozen" turbulence hypothesis: i.e. assuming that the variability in the measured surface radiation was dominated by the advection of the spatial structures of the cloud fields across the local point of the observation rather than their temporal local variability (see above).

Figures 16-18 show power spectra of the global transmittance (vs. the horizontal wavenumbers $k$ ) for the broken-cloud, cirrus and overcast sky conditions respectively. The spectral data were taken from the Fig. 5 of 
the Ref. 27. The dashed curves in the Figs. 16, 17 indicate correspondence to the spectrum Eq. (22) whereas the dashed curve in the Fig. 18 (overcast cloudy sky)

indicates correspondence to the spectrum Eq. (15).
[1] N. Ohtomo, K. Tokiwano, Y. Tanaka et. al., J. Phys. Soc. Jpn. 641104 (1995)

[2] U. Frisch and R. Morf, Phys. Rev., 23, 2673 (1981)

[3] J.D. Farmer, Physica D, 4, 366 (1982)

[4] A. Brandstater and H.L. Swinney, Phys. Rev. A 35, 2207 (1987)

[5] D.E. Sigeti, Phys. Rev. E, 52, 2443 (1995)

[6] A. Bershadskii, EPL, 88, 60004 (2009)

[7] J.E. Maggs and G.J. Morales, Phys. Rev. Lett., 107, 185003 (2011); Phys. Rev. E 86, 015401(R) (2012)

[8] D.A. Donzis K.R. Sreenivasan, P.K. Yeung, Flow Turbulence Combust., 85, 549 (2010)

[9] A. Kumar, A.G. Chatterjee and M.K. Verma, Phys. Rev. E, 90, 023016 (2014)

[10] E. Levich and A. Tsinober, Phys. Lett. A 93, 293 (1983)

[11] H.K. Moffatt and A. Tsinober, Annu. Rev. Fluid Mech., 24, 281 (1992)

[12] A. Bershadskii and A. Tsinober, Phys. Rev. E, 48, 282 (1993)

[13] D. Finn, B. Lamb, M. Y. Leclerc, S. Lovejoy, S. Pecknold, and D. Schertzer, J. Appl. Meteorol. 40, 229 (2001)

[14] A. S. Monin, A. M. Yaglom, Statistical Fluid Mechanics, Vol. II: Mechanics of Turbulence (Dover Pub. NY, 2007)

[15] M.Y. Leclerc, N. Meskhidze and D. Finn, Agricultural and Forest Meteorology, 117, 145 (2003)

[16] S.-Ya Ogino, M.D. Yamanaka, S. Kaneto, T. Yamanouchi and S. Fukao, Proc. NIPR Symp. Polar Meteorol. Glaciol., 11, 199 (1997)

[17] K. Wargan, S. Pawson, I. Stajner, and V. Thouret, J. Geophys. Res., 115, D24316 (2010)

[18] D. C. Johnston, Phys. Rev. B, 74, 184430 (2006).

[19] U. Rummel, C. Ammann, A. Gut, F. X. Meixner, and M.O. Andreae, J. Geophys. Res., 107(D20), 8050, (2002)

[20] D.M.D. Hendriks, J. van Huissteden, A.J. Dolman, and M.K. van der Molen, Biogeosciences, 4, 411 (2007)

[21] E. Yee, P.R. Kosteniuk, G.M. Chandler, C. A. Biltoft, and J.F. Bowers. Boundary-Layer Meteorology, 65, 69 (1993)

[22] A. Detwiler and A.J. Heymsfield, J. Atmos. Sci., 44, 1899 (1987)

[23] L.N. Fletcher, G.S. Orton and J.H. Rogers, Icarus, 286, 94 (2017).

[24] R.G. Cosentino, A. Simon, and R. Morales-Juberias, J. Geophys. Res.: Planets, 124, 1204 (2019)

[25] P. Patra, K., Santhanam, M. S., Manimaran, P., Takigawa, M., and T. Nakazawa, arXiv:nlin/0610038 (2006)

[26] R. Botha, C. Labuschagne, A.G. Williams, G. Bosman, E.-G. Brunke, A. Rossouw and R. Lindsay, Atmos. Environ., 176, 30 (2018)

[27] B.L. Madhavan, H. Deneke, J. Witthuhn, and A. Macke, Atmos. Chem. Phys., 17, 3317 (2017) 